\title{
A highly efficient and tumor vascular-targeting therapeutic technique with size-expansible gadofullerene nanocrystals
}

\author{
Mingming Zhen ${ }^{1}$, Chunying Shu ${ }^{1}$, Jie $\mathrm{Li}^{1}$, Guoqiang Zhang ${ }^{1}$, Taishan Wang ${ }^{1}$, Yi Luo ${ }^{2}$, Toujun Zou ${ }^{1}$, \\ Ruijun Deng ${ }^{1}$, Fang Fang ${ }^{3}$, Hao Lei ${ }^{3}$, Chunru Wang ${ }^{1^{*}}$ and Chunli Bai ${ }^{1}$
}

\begin{abstract}
It has long been a dream to achieve tumor targeting therapy that can efficiently reduce the toxicity and severe side effects of conventional antitumor chemotherapeutic agents. Taking advantage of the abnormalities of tumor vasculature, we demonstrate here a new powerful tumor vascular-targeting therapeutic technique for solid cancers that applies advanced nanotechnology to cut off the nutrient supply of tumor cells by physically destroying the abnormal tumor blood vessels. Water soluble magnetic Gd@ $\mathrm{C}_{82}$ nanocrystals of the chosen sizes are deliberately designed with abilities to penetrate into the leaky tumor blood vessels. By triggering the radiofrequency induced phase transition of gadofullerene nanocrystals while extravasating the tumor blood vessel, the explosive structural change of nanoparticles generates a devastating impact on abnormal tumor blood vessels, resulting in a rapid and extensive ischemia necrosis and shrinkage of the tumors. This unprecedented target-specific physiotherapy is found to work perfectly for advanced and refractory solid tumors.
\end{abstract}

\section{INTRODUCTION}

Solid tumors possess unique pathophysiological vasculatures that cannot be found in normal tissues or organs [1], including the hyper vasculatures, the defective vascular architecture, the lack of lymphatic drainage and the extensive production of a number of permeability mediators [2-5]. These characters are known as the enhanced permeability and retention (EPR) effects that have been widely used to enhance the tumor selectivity of antitumor nanomedicines [6-8]. It was reported that with time the concentration of some intravenously (IV) administered nanosized anticancer drugs at the tumors could become several folds higher than that in normal tissues $[9,10]$. Naturally, the central focus of current studies has been on how to increase the retention and accumulation of nanoparticles in tumor vasculatures for better tumor targeting therapy and imaging [11-13]. Although the extravasation mechanism of nanomaterials is not fully understood yet due to the endothelial biodiversity, it has been observed that the permeability of tumor blood vessels can indeed be affected by the nanoparticles of different sizes and shapes in many theoretical and experimental studies [14,15].

\section{EXPERIMENTAL SECTION}

\section{Preparation of gadofullerene nanocrystals}

The water-soluble gadofullerene nanocrystals (GFNCs) were prepared through a one-pot reaction. Simply, the suspension of 1000 mg Gd@C $\mathrm{C}_{82}$ (99\% purity, as obtained from Xiamen Funano Co.) in $30 \%$ of aqueous $\mathrm{H}_{2} \mathrm{O}_{2}(70 \mathrm{~mL})$ and $10 \% \mathrm{NaOH}(30 \mathrm{~mL})$ was vigorously stirred at $50^{\circ} \mathrm{C}$ under ambient condition for $3 \mathrm{~h}$. The black suspension was gradually dissolved and turned into a yellow solution with slight insoluble materials. A transparent yellowish solution was obtained after filtration. Then $100 \mathrm{~mL}$ ethanol was added to the concentrated solution (ca. $10 \mathrm{~mL}$ ) to precipitate the solutes. The suspension was centrifuged at $3018.6 \times \mathrm{g}$ for $5 \mathrm{~min}$ and the supernatant was discarded. The obtained yellowish precipitates were washed twice with ethanol (ca. $100 \mathrm{~mL}$ ) and dried under vacuum at $50^{\circ} \mathrm{C}$ overnight, producing the hydroxyl bearing water-soluble GFNCs.

\section{The $200 \mathrm{MHz}$ radiofrequency (RF) generator}

There are two ways to utilize $200 \mathrm{MHz}$ RF with the power

\footnotetext{
${ }^{1}$ Beijing National Laboratory for Molecular Sciences, Key Laboratory of Molecular Nanostructures and Nanotechnology, Institute of Chemistry, Chinese Academy of Sciences, Beijing 100190, China

${ }^{2}$ Bio-X Division, Hefei National Laboratory for Physical Sciences at the Microscale and Synergetic Innovation Center of Quantum Information \& Quantum Physics, University of Science and Technology of China, Hefei 230026, China

${ }^{3}$ National Center for Magnetic Resonance in Wuhan, State Key Laboratory of Magnetic Resonance and Atomic and Molecular Physics, Wuhan Institute of Physics and Mathematics, Chinese Academy of Sciences, Wuhan 430071, China

* Corresponding author (email: crwang@iccas.ac.cn)
} 
of $100 \mathrm{~W}$. The first is the standard equipped $200 \mathrm{MHz}$ RF generator (BioSpec 4.7/30) in the $4.7 \mathrm{~T}$ commercial magnetic resonance imaging (MRI). The tumor-bearing mice could be placed in the center of the magnet tube and a body coil. For conveniently making some comparing experiments, we have constructed a second $200 \mathrm{MHz}$ RF generator included commercial MXG RF Analog Signal Generator (100 kHz-1 GHz, N5181A, Agilent), signal amplifier BLAXH 20 (Bruker) and a home-made helical antennas. Its power could easily reach to $100 \mathrm{~W}(10 \mathrm{~Hz}$ pulse frequency, $10 \%$ duty cycle).

\section{Differential scanning calorimetry (DSC) measurements}

DSC (Q2000, Waters Company, USA) was used to study the phase transition temperature of bulk $\mathrm{Gd} @ \mathrm{C}_{82}$ with a heating rate of $10^{\circ} \mathrm{C} \mathrm{min}^{-1}$. The water solution DSC thermograms were obtained using a VP-DSC microcalorimetric system. The GFNCs solutions were degassed for $10 \mathrm{~min}$, and then transferred into a Tantaloy $61 \mathrm{~T}$ Malloy sample cell of $500 \mu \mathrm{L}$. The sample cell containing the solution and the reference cell with water were cooled down to $10.0^{\circ} \mathrm{C}$ before the temperature scan started. The solution DSC thermograms were recorded at a heating rate of $30^{\circ} \mathrm{C} \mathrm{h}^{-1}$.

\section{Animal models}

All animal operations were in accordance with the institutional animal use and care regulations, the Experimental Animal Center of Wuhan Institute of Physics and Mathematics, Chinese Academy of Sciences (CAS). BALB/c nude mice and $\mathrm{BALB} / \mathrm{c}$ mice $(n=3-5)$ weighing $18-20 \mathrm{~g}$ were purchased from the Zhongnan Hospital of Wuhan University (Wuhan, China). Five-week-old female mice were injected on the right rear flank area with $5 \times 10^{6}$ tumor cells (H22 mouse hepatoma cancer cells and 4T1 mouse mammary cancer cells) in $100 \mu \mathrm{L}$ of serum free medium. The treatments were conducted after 5-7 days after the tumor inoculation.

\section{MRI}

In vivo MRI measurements were performed using a $4.7 \mathrm{~T}$ instrument with a bore diameter of $6 \mathrm{~mm}$ (PharmaScan, Bruker BioSpin, Germany). Athymic nude mice bearing H22 liver tumors were anesthetized using 2\% isoflurane, and the tumors were positioned at the center of the RF coil (200 MHz). The physiologic conditions of the animals were monitored using a respiratory monitoring device during the scanning. After a pilot scans for determining the region of interest, a multislice spin-echo sequence was used with a repetition time (TR) of $400 \mathrm{~ms}$, echo time (TE) of $11 \mathrm{~ms}$, and slice thickness of $1 \mathrm{~mm}$. Mice were IV injected
$10 \mathrm{mmol} \mathrm{L}^{-1}$ GFNCs solution and treated under RF pulse continuously for $0.5-1 \mathrm{~h}$, and acquired $\mathrm{T}_{1}$-weighted images before and after injection in a 10 min interval.

\section{Magnetic resonance angiography (MRA)}

MRA was performed on a $7.0 \mathrm{~T}$ scanner (BioSpec 7.0 $70 / 20$, Bruker, German) using a mice body coil to acquire signals. MRA images were acquired using TOF-2D-FLASH sequence with parameters as follows: $\mathrm{T}_{1}$-weighted fast field echo (T1FFE), TR $=12 \mathrm{~ms}, \mathrm{TE}=2 \mathrm{~ms}$, field of view (FOV) $=40 \mathrm{~mm} \times 40 \mathrm{~mm}$, number of slices $=100$, slice thickness $=0.3 \mathrm{~mm}$, flip angle $=50^{\circ}$. The pseudo MRA images were analyzed using data software (Mricron).

\section{Optical imaging}

Luciferase was used as a marker in bioluminescence images to evaluate the growth of breast tumors after GFNCs and RF treatments by the CRI Maestro Imaging System (USA). The mice were anesthetized by intraperitoneal (IP) injection of $100 \mu \mathrm{L}$ pelltobarbitalum natricum (1\%). Anesthetized mice were IP injected with $75 \mathrm{mg} / \mathrm{kg}$ D-luciferin (Caliper Life Sciences, USA) in phosphate buffered saline (PBS). After injection ca. $10 \mathrm{~min}$, bioluminescence images were acquired using optical imaging and the acquisition time was $0.1 \mathrm{~s}$. Images were set at the indicated pseudo color scale to show the relative bioluminescent changes over time.

\section{Environmental scanning electron microscopy (ESEM) observation of the blood vessels of tumors}

The fresh tumor tissues after treatment by GFNCs and RF were obtained from surgically explanted samples, and after passage in $2.5 \%(v / v)$ glutaraldehyde, they were sectioned with a scalpel in $2 \mathrm{~mm} \times 1 \mathrm{~mm}$ samples with parallel surfaces to get a flat surface of observation. The images were observed with the SEM-JSM-6301F (JEOL, Japan).

\section{Histopathology assay}

Hematoxylin and eosin (H\&E) staining was analyzed following the standard protocol. Briefly, after the mice were treated by GFNCs combined with RF and sacrificed, the tumors were excised and fixed in formalin and embedded in paraffin. After washing with flowing water for $5 \mathrm{~min}$, the slides were treated with gradient concentrations of alcohol (100\%, 95\%, 80\% and 70\%), each for $10 \mathrm{~min}$. The hematoxylin staining was performed for about $7 \mathrm{~min}$ and washed with water for $5 \mathrm{~min}$. The eosin staining was performed for about $3 \mathrm{~min}$. The slides were washed, treated with xylene, and mounted with resinene. Then the stained slices were observed by a Nikon eclipse E400 microscope (Nikon 
France SA).

\section{Biochemical measurements}

Blood samples were harvested by quickly removing the eyeball after treatments. Whole blood was placed in a clotted vial and centrifuged, and serum was collected for biochemical analysis. The toxicity towards liver and kidney after GFNCs and RF treatment was evaluated with serum levels of total, alanine aminotransferase (ALT), aspartate aminotransferase (AST), bilirubin levels (TBIL), blood urea nitrogen (BUN), creatinine (Cr) and uric acid (UA) using a biochemical autoanalyzer (Type 7170, Hitachi, Japan).

\section{${ }^{131}$ I radiolabeled biodistribution}

GFNCs (20 mg) was dissolved in $2 \mathrm{~mL}$ PBS solution ( $\mathrm{pH} 7$ ) and then $\mathrm{Na}^{131} \mathrm{I}(5.0 \mathrm{MBq}, 20 \mu \mathrm{L})$ in distilled water was added to the solution, immediately followed by the addition of chloramine-T solution $\left(3 \mathrm{mg} \mathrm{mL} \mathrm{mL}^{-1}, 20 \mu \mathrm{L}\right)$ dissolved in sodium phosphate buffer $\left(50 \mathrm{mmol} \mathrm{L}^{-1}, \mathrm{pH}\right.$ 7.4). After incubation for $10 \mathrm{~min}$, a sodium bisulfite solution $(0.1 \mathrm{mg} / 0.05$ $\mathrm{mL})$ dissolved in sodium phosphate buffer $\left(50 \mathrm{mmol} \mathrm{L} \mathrm{L}^{-1}\right.$, $\mathrm{pH}$ 7.4) was added into the reaction solution. Sephadex G-25 column was employed to separate ${ }^{131}$ I-GFNCs from free ${ }^{131} \mathrm{I}$, and the radiochemical purity was determined by a double paper chromatography using chromatography paper strips (Whatman, $1.5 \mathrm{~cm} \times 15 \mathrm{~cm}$ ). The major organs (the heart, liver, spleen, lung, kidney, bone, muscle and tumor) were collected and weighed at $1 \mathrm{~h}, 4 \mathrm{~h}, 24 \mathrm{~h}, 48 \mathrm{~h}$, $10 \mathrm{~d}$, and $30 \mathrm{~d}$ post-injection, respectively. After nitrolysis, the radioactivity was scanned on an automatic thin layer chromatography scanner (BIOSCAN, Washington DC, USA).

\section{RESULTS}

\section{Size-expansible phase transition of RF-assisted GFNCs}

Our laboratory has been devoted to the study of fullerene and metallofullerene nanomaterials in the last decades $[16,17]$, in particular the metallofullerene particles in different forms [18-21]. As motivated by a recent study on the synthesis of water soluble $\mathrm{Gd} @ \mathrm{C}_{60}$ crystalline particles with a simple solid-liquid reaction [22], we prepared similar crystalline $\mathrm{Gd} @ \mathrm{C}_{82}(\mathrm{OH})_{m}$ nanoparticles using this novel technique. The purity of the sample was verified by both matrix-assisted laser desorption/ionization time-offlight mass spectrometry (MALDI-TOF MS) and high performance liquid chromatography (HPLC) measurements before using (Fig. S1). The $\mathrm{Gd@C} \mathrm{C}_{82}$ sample was dried up in air, and the bulk Gd@C $\mathrm{C}_{82}$ powder was treated with $\mathrm{H}_{2} \mathrm{O}_{2}$ and $\mathrm{NaOH}$ to form $\mathrm{Gd} @ \mathrm{C}_{82}$ nanocrystals with many hy- droxyl groups conjugating on the surface, as illustrated in Fig. 1a and labeled as $\left(\mathrm{Gd} @ \mathrm{C}_{82}\right)_{m}(\mathrm{OH})_{n}$ afterwards.

The water soluble $\left(\mathrm{Gd} @ \mathrm{C}_{82}\right)_{m}(\mathrm{OH})_{n}$ was characterized by various spectrometry. The cryo-transmission electron microscopy (Cryo-TEM) as shown in Fig. 1b reveals that the gadofullerenes are spherical or ellipsoid particles with an average size of ca. $140 \mathrm{~nm}$, which is larger than previously reported gadofullerenes derivatives [22,23]. Selected-area electron diffraction (SAED) of the sample shows clear Debye rings, suggesting that the GFNCs are indeed composed of multiple Gd@ $\mathrm{C}_{82}$ molecules in the core and with hydroxyl groups attached on the particle surface. The GFNCs sample was further characterized by X-ray photoelectron spectroscopy (XPS). As shown in Fig. S2 and Table $\mathrm{S} 1$, the composition of the materials was found to contain mainly $\mathrm{C}, \mathrm{O}, \mathrm{Gd}$, as well as a little $\mathrm{Na}$ that is possibly from the reactant $\mathrm{NaOH}$ to form a week complex with poly-ion $\mathrm{Na}^{+}$salt [24].

Thecrystallinegadofullerenes, e.g., $\mathrm{Gd} @ \mathrm{C}_{60}\left[\mathrm{C}(\mathrm{COOH})_{2}\right]_{10}$ and $\mathrm{Gd} @ \mathrm{C}_{60}(\mathrm{OH})_{x}[22],\left(\mathrm{Gd} @ \mathrm{C}_{82}\right)_{m}(\mathrm{OH})_{n}$ and others [25], have been found to own a common interesting physical property, namely, a size-expansible phase transition under heating. As illustrated in Figs $1 \mathrm{c}$ and d, we observed that the bulk Gd@C $\mathrm{C}_{82}$ polycrystals experience a conversable phase transition at ca. $344 \mathrm{~K}\left(71^{\circ} \mathrm{C}\right)$, while the water soluble GFNCs go through an irreversible phase transition at $354 \mathrm{~K}\left(81^{\circ} \mathrm{C}\right)$. Interestingly, due to the superparamagnetic property of the gadofullerene material (Fig. 1e), the phase transition of this material can also be achieved by applying an RF irradiation for several minutes (Fig. S3). As revealed by the dynamic light scattering (DLS) measurements (Fig. 1f), after treating $1 \mathrm{mmol} \mathrm{L}^{-1}$ GFNCs solution by $200 \mathrm{MHz}$ RF for $30 \mathrm{~min}$, the size of GFNCs is found to expand from $136 \pm 7.1$ to $204 \pm 10.6 \mathrm{~nm}$ as the result of the RF induced phase transition.

\section{The anticancer mechanism of RF-assisted GFNCs}

The GFNCs material was originally taken as MRI contrast agent, and a group of $5 \mathrm{H} 22$ tumor-bearing athymic nude mice were taken as test animals. The tumor-bearing mice were IV administered with $150 \mu \mathrm{L}$ of $10 \mathrm{mmol} \mathrm{L}^{-1}$ water-soluble GFNCs and after $10 \mathrm{~min}$, this MRI contrast agent was tested for tumor diagnosis using a $4.7 \mathrm{~T}$ small animal MRI with a $200 \mathrm{MHz}$ resonance frequency (BioSpec 4.7/30). Each mouse was put in the center of the magnet tube and a $200 \mathrm{MHz}$ of pulsed RF was continuously irradiated to the mouse for $30 \mathrm{~min}$ to induce the phase transition of GFNCs. Remarkably, all the 5 mice treated by RF-assisted GFNCs were found to experience a rapid tumor color darkening in 2-4 h. The real-time morphological changes in tumor area 
a
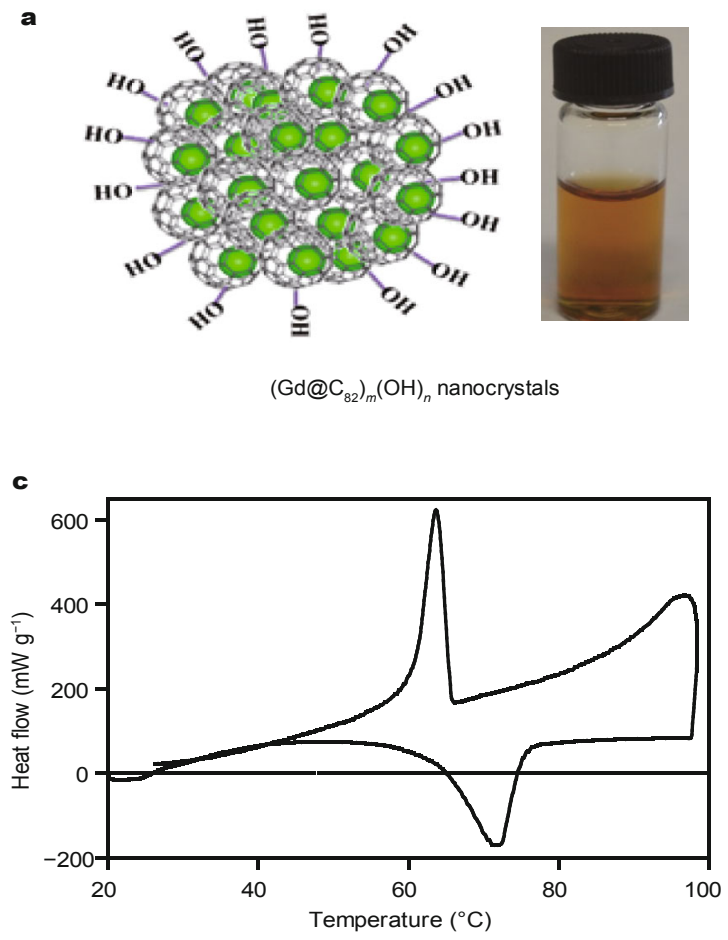

e

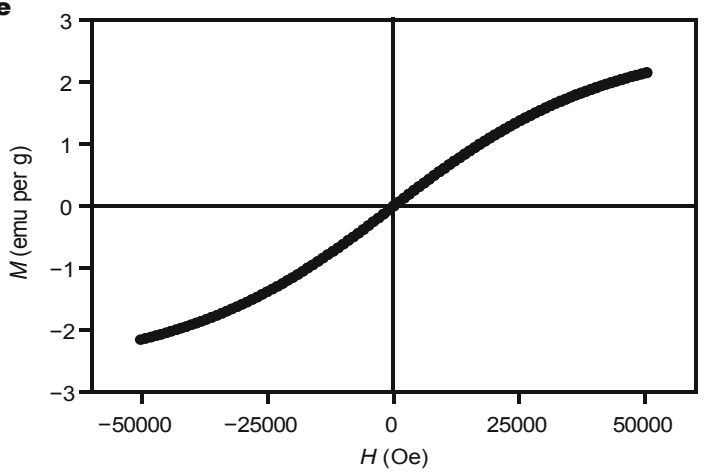

b

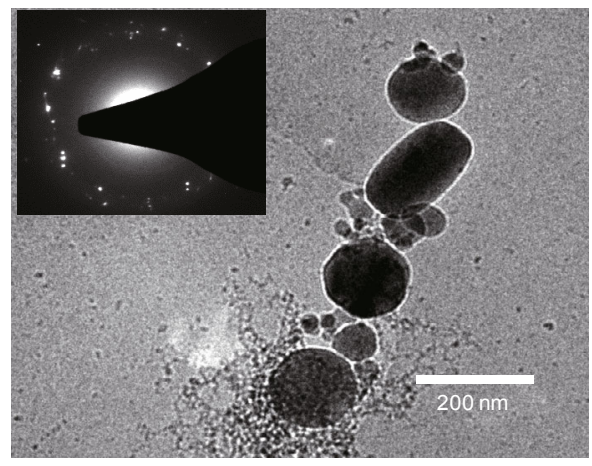

d

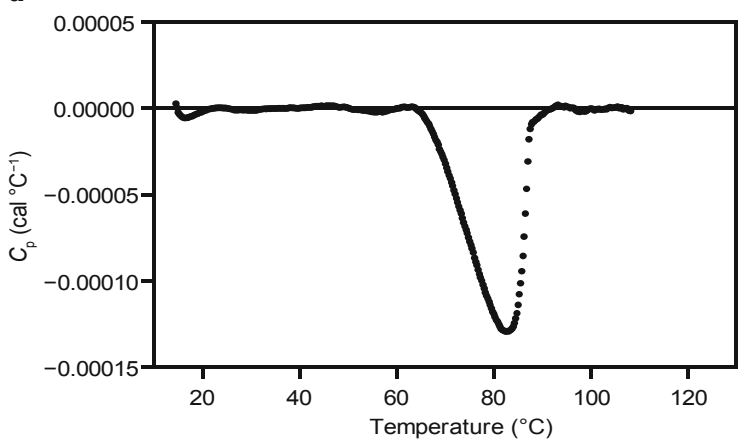

f

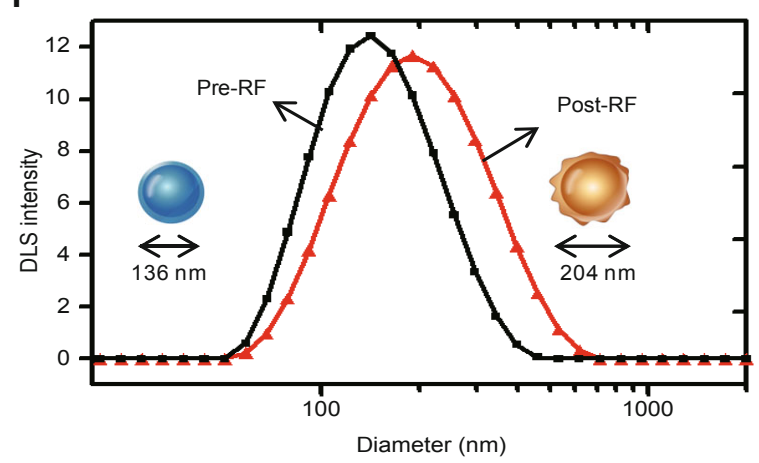

Figure 1 Characterizations of the GFNCs and the size expansion after RF irradiation. (a) As-synthesized GFNCs aqueous solution and the schematic diagram of functionalized GFNCs by hydroxylation with $\mathrm{H}_{2} \mathrm{O}_{2}$ in alkaline. (b) Cryo-TEM micrograph of GFNCs. The inset is the SAED pattern, in which the Debye ring demonstrates the crystalline form of GFNCs. (c) Powder DSC measurement for bulk Gd@ $\mathrm{C}_{82}$ with the phase transition temperature at ca. $344 \mathrm{~K}\left(71^{\circ} \mathrm{C}\right)$. (d) Solution DSC measurement for GFNCs with $1 \mathrm{mmol} \mathrm{L}^{-1} \mathrm{Gd}^{3+}$ concentration with the phase transition temperature at ca. $354 \mathrm{~K}\left(81^{\circ} \mathrm{C}\right)$. (e) The magnetization vs. field (M-H) curve of GFNCs. No hysteresis loop exists in the curve, indicating the superparamagnetism of the particles. (f) Average hydrodynamic diameter of GFNCs increases from $136( \pm 7.1)$ to $204( \pm 10.6) \mathrm{nm}$ under the radiation of $200 \mathrm{MHz} \mathrm{RF}$ for $30 \mathrm{~min}$.

were monitored by MRI within 90 min of the treatment (Fig. 2). The pseudo MR images were analyzed using data software (MRIcron) to objectively quantify the ratio of hypointensity volume to whole tumor. It was clearly observed that the tumor began to form hypointensity area after only 30 min treatment, and the ratio was gradually increased to ca. $8 \%$ after $90 \mathrm{~min}$ of RF irradiation (Figs 2b-d). After RF-assisted GFNCs treatment, the tumor was observed to induce a rapid and extensive necrosis, and finally serious inner collapses occurred in $24 \mathrm{~h}$ (Fig. S4).

Since magnetic hyperthermia was widely studied for tumor treatments [26], and the tumor ablation therapeutic technique by RF-induced heating of some charged particles has been clinically used recently [27-29], the anticancer mechanism of the RF-assisted GFNCs was primarily proposed as a magnetic hyperthermia effect. However, a series of deliberately designed experiments were performed that completely rule out this mechanism. 


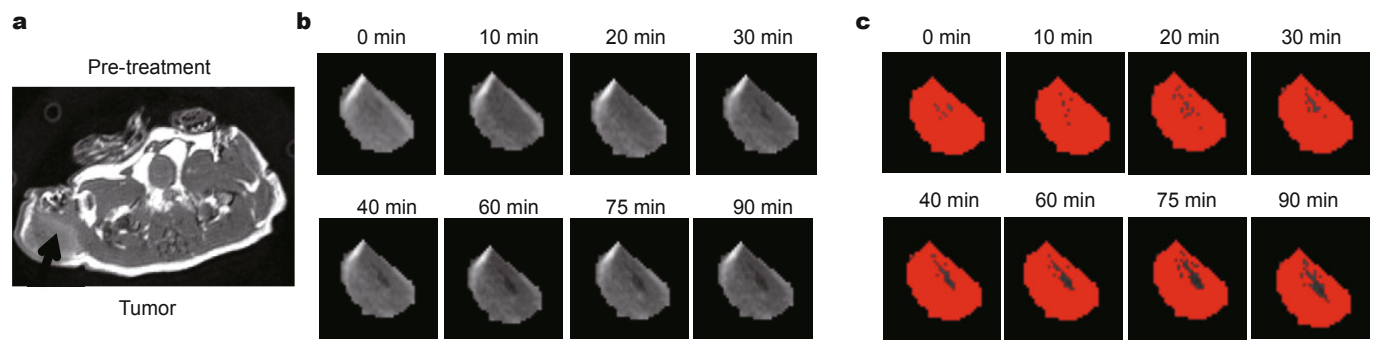

d

\begin{tabular}{|l|c|c|c|c|c|c|c|c|}
\hline Time (min) & 0 & 10 & 20 & 30 & 40 & 60 & 75 & 90 \\
\hline$H / W \%$ & 1.6 & 2.7 & 2.8 & 3.7 & 5.4 & 6.4 & 7.4 & 8.0 \\
\hline
\end{tabular}

Figure 2 The dynamical MRI of tumor by 90 min RF-assisted GFNCs treatments. (a) The MR images of liver tumor-bearing mice pre-treatment. (b) The interception MRI of tumor area. (c) The pseudo MR images were analyzed using data software (Mricron). (d) The quantitative ratio of hypointensity volume $(H)$ in the whole tumor volume $(W)$.

First, around $10 \mathrm{~mL}$ of $1 \mathrm{mmol} \mathrm{L}^{-1}$ GFNCs solution was applied on $200 \mathrm{MHz}$ RF irradiation for $30 \mathrm{~min}$, but it was observed that the solution underwent only a moderate temperature change, i.e., the temperature increased only slightly from ca. 20.8 to $23.4^{\circ} \mathrm{C}$ during this process, which was in the same level with those of $\mathrm{NaCl}$ and $\mathrm{Gd}$-diethylenetriaminepentaacetic acid (DTPA) groups (Fig. 3a).
Second, cell incubation with GFNCs experiments by three kinds of cells, i.e., mice hepatic cancer cell H22, human hepatic cancer line HepG2, and mice embryonic liver cell BNL (normal cells) were performed under RF irradiation, respectively. The results indicate that all these cells maintain high viability after long time of RF irradiation with GFNCs (Figs 3d-f).
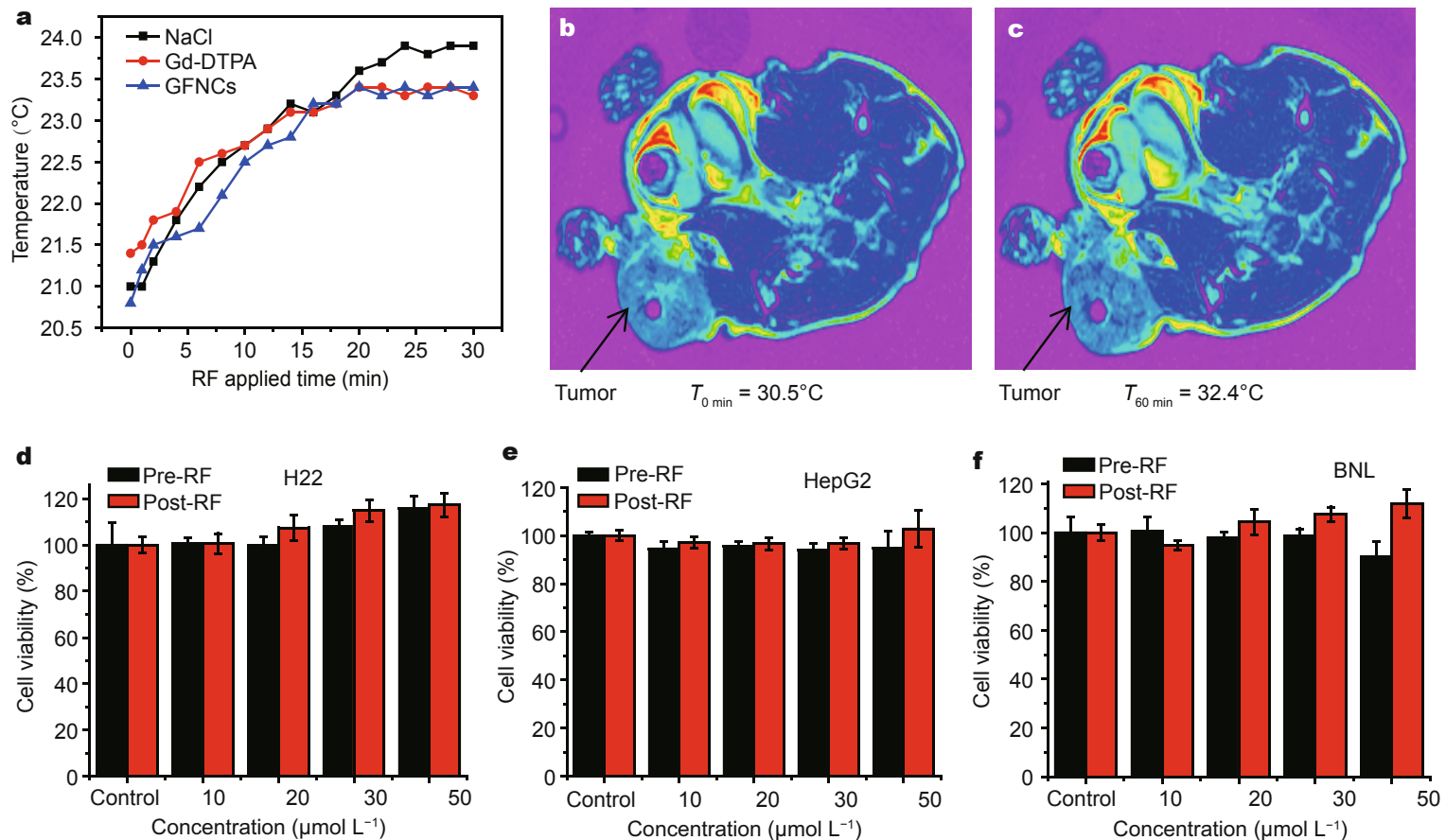

Figure 3 The evidences of excluding the hyperthermia effects. (a) Temperature changes of $0.9 \% \mathrm{NaCl}, 1 \mathrm{mmol} \mathrm{L}^{-1} \mathrm{Gd}-\mathrm{DTPA}$ and $1 \mathrm{mmol} \mathrm{L}{ }^{-1} \mathrm{GFNCs}$ solution exposed under $200 \mathrm{MHz}$ RF. (b and c) The temperature changes of tumor tissues monitored by an optical fiber temperature sensor during the treatment. (d-f) The cell viabilities of three kinds of cells incubated with GFNCs pre- and post-RF irradiations: (d) mice hepatic cancer cell H22; (e) human hepatic cancer line HepG2; (f) mice embryonic liver cell BNL (normal cells). The cells were incubated with $10-50 \mu \mathrm{mol} \mathrm{L}^{-1}$ GFNCs. The results indicate that GFNCs exhibit nontoxicity in vitro after RF irradiation. 
Third, in order to completely exclude the possibility of magnetic hypothermia effects in current therapeutic experiments, an optical fiber temperature sensor was employed to directly measure the temperature of tumor section during the RF-assisted GFNCs treatment. As shown in Figs $3 \mathrm{~b}$ and $\mathrm{c}$, the tumor-bearing mouse was placed under the constant temperature cushion $\left(37^{\circ} \mathrm{C}\right)$, and the optical fiber was inserted on the center area of the tumor. Fascinatingly, it was observed that the temperature of tumor area increased only about $2^{\circ} \mathrm{C}$ during $60 \mathrm{~min} 200 \mathrm{MHz} \mathrm{RF}$ irradiation. Furthermore, we reproduced the $200 \mathrm{MHz}$ RF generator (Fig. S5) in laboratory to simulate that used in the 4.7 T MRI machine, thus we were able to use an infrared thermal camera to monitor the temperature change during RF-assisted GFNCs treatment (Fig. S6). Once again, less than $2^{\circ} \mathrm{C}$ temperature increase was measured in the tumor area after $1 \mathrm{~h}$ of RF irradiation.

After excluding the magnetic hyperthermia mechanism, we performed a series of experiments trying to find out the reason that induces the rapid tumor necrosis. Finally, since the ESEM study of the tumor tissue showed extensive impaired blood vessels inside the tumor body, a clue for the tumor therapy was revealed that the RF-assisted GFNCs may destroy the tumor vascular system. As shown in the ESEM images of the tumor blood vessels of H22 liver tumor (Figs $4 \mathrm{a}-\mathrm{c}$ ), extensive damages with only the basement membrane were observed after the RF-assisted GFNCs treatment, indicating that large scales of endothelial cells (ECs) were chipped off during the therapy process.
Undoubtedly, the damage of tumor blood vessels must induce a rapid reducing of blood flow to tumor area or even cutting it off, so the MRA was applied to monitor the blood flow in tumor area which is a powerful technique to show blood flow of the tested animals. As shown in Figs $4 \mathrm{~d}-\mathrm{g}$, the MRA images of RF-assisted GFNCs exhibit identifiable reduction of the arterial vessels flowing to the tumor (Figs $4 \mathrm{f}$ and $\mathrm{g}$ ), which is clearly contrast to the unchanged blood vessels of the untreated group (Figs $4 \mathrm{~d}$ and e).

Based on the above observation, a new anticancer mechanism through destroying tumor vascular system by the RF-assisted GFNCs was presented, i.e., the RF-assisted GFNCs material attacks the tumor vascular structures during the extravasation process, and the loss of nutrient from the impaired vessels is responsible for the observed necrosis and shrinkage of the tumors.

The therapeutic mechanism is schematically summarized in Fig. 5. After the water soluble GFNCs IV injecting into the tumor-bearing mice, the particles would rapidly flow along the normal blood vessels to reach the tumor sites. They can force themselves to penetrate the leaks around endothelia cells, as the consequence of the EPR effect. It has been estimated that the GFNCs would spend several to dozens of minutes to permeate the blood vessels depending on their size. When the RF is applied, the GFNCs can undergo a phase transition. The explosive volume expansion accompanying with the possible rotation of the magnetic nanocrystals can thus destroy the networks of endothelia cells and abscission from the basilemma. The
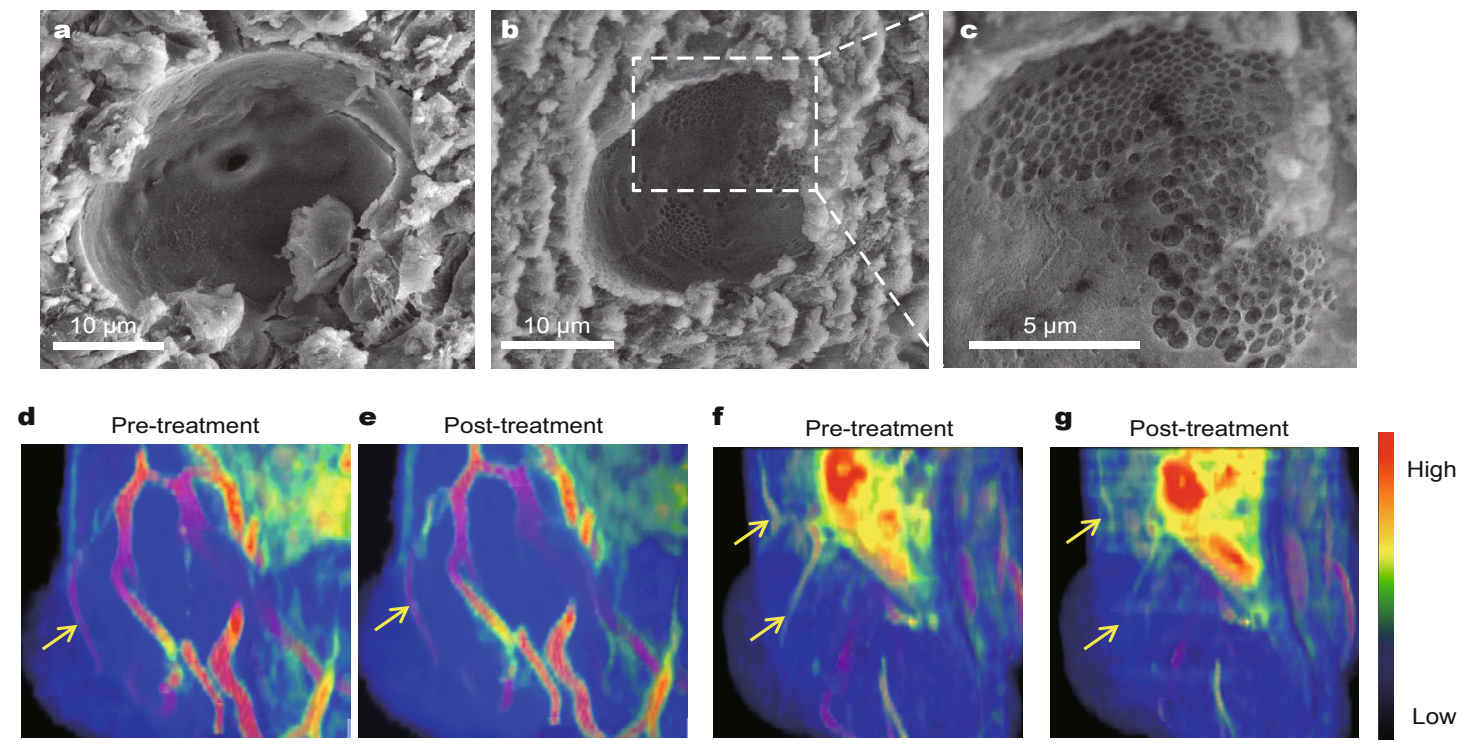

Figure 4 The observation of tumor blood vessels. (a-c) ESEM microphotography of tumor blood vessels treated (a) without and (b, c) with RF-assisted GFNCs. The endothelial cells were chipped off from the basilemma at $24 \mathrm{~h}$ after the treatment. $(\mathrm{d}-\mathrm{g}$ ) The MRA images of tumor vascular (yellow arrows) for (d) pre- and (e) $1.5 \mathrm{~h}$ post of control groups, and (f) pre- and (g) $1.5 \mathrm{~h}$ post RF-assisted GFNCs treatments. 


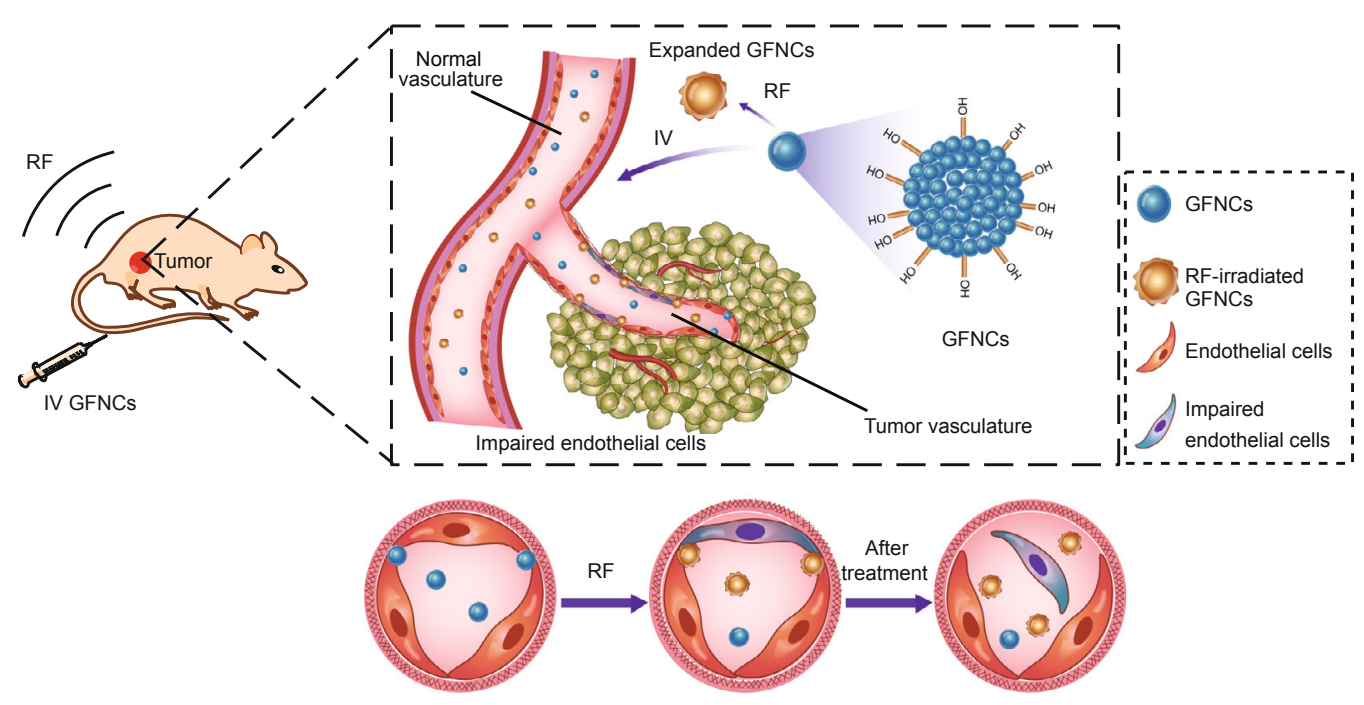

Figure 5 A schematic draw of the antitumor mechanism of RF-assisted GFNCs treatments. The GFNCs are first accumulated in the lining of the tumor blood vessels due to EPR effect, and then the sudden volume expansion and the rotation of the RF-assisted GFNCs induce the exfoliation of endothelia cells.

impaired vessels cut off the nutrient supply of tumor cells, leading to an accelerated ischemic necrosis of the tumors. It is noted that this function is similar to what the vascular disrupting agents (VDAs) have, namely to alter the shape of the endothelial cell by disrupting the cytoskeleton and cellto-cell junctions [30,31]. However, the RF-assisted GFNCs offers a physio-therapeutic technique that relies on the inherent pure physical properties of tumor vasculatures to avoid uncontrollable biochemical response introduced by conventional VDAs [32].

We have designed and performed a series of comparing experiments that unambiguously convinced this hypothesis. First, several control experiments were carried out to show that both RF and the as-prepared GFNCs were necessary for the tumor therapy. As shown in Fig. S7, six parallel tests on following H22-bearing mice were carried out: (a) the as-prepared GFNCs were IV injected and $200 \mathrm{MHz}$ RF applied; (b) the same as (a) but without RF; (c) the GFNCs were irradiated by RF for 30 min in vitro then IV injected and $200 \mathrm{MHz}$ RF applied; (d) the GFNCs were heated to $80^{\circ} \mathrm{C}$, cooled down to room temperature, then IV injected and $200 \mathrm{MHz}$ RF applied; (e) GFNCs were directly injected into the tumor under RF, and $200 \mathrm{MHz}$ RF applied; (f) $\left(\mathrm{C}_{60}\right)_{m}(\mathrm{OH})_{n}$ were IV injected and $200 \mathrm{MHz} \mathrm{RF}$ applied. It is obvious that both the as-prepared GFNCs and the 200 $\mathrm{MHz} \mathrm{RF}$ are indispensible to realize the tumor therapy. Especially, for those GFNCs after heating or RF irradiating treatments, they nearly lose their antitumor effects in animal tests (Figs S7c and d) due to the lack of RF-induced phase transition.

Fascinatingly, the therapy mechanism indicates that the GFNCs must be IV injected into the mice, otherwise the GFNCs could not be embedded in tumor vascular gaps to strip off the endothelia cells while they were excited by the RF. Therefore, it is natural to understand that no sign of antitumor activity can be observed while the GFNCs being directly injected into the tumor tissues, since the size expansion of GFNCs is harmless to the tumor vasculatures in this situation. Moreover, the similar size of the as-prepared crystalline $\mathrm{C}_{60}$ nanoclusters do not demonstrate any antitumor activity as they could not be triggered by the applied $\mathrm{RF}$ to occur explosive phase transition (Fig. S7f).

\section{The universal tumor therapy in vivo by RF-assisted GFNCs}

System studies of the antitumor efficacy in vivo have been performed by MRI spectrometry, bioluminescence microscopy, and histology analysis, following the working procedure given in Fig. 6a. MRI spectrometry is particularly useful, since it is able to study the retention property of the paramagnetic GFNCs and simultaneously provides the RF needed for the GFNC phase transition. The MRI micrographs clearly illustrate an extensive tumor necrosis $2-4 \mathrm{~h}$ after the treatment (Fig. 6b), accompanying with the tumor color changing gradually from pink to black by ischemia necrosis at $6 \mathrm{~h}$, and finally the tumor was observed to coagulate and regress after $24 \mathrm{~h}$ (Figs $6 \mathrm{c}$ and d). Moreover, after 15 days of the treatment, the tumors were nearly necrosis with only a crustlike scab remaining, in contrast to 
a

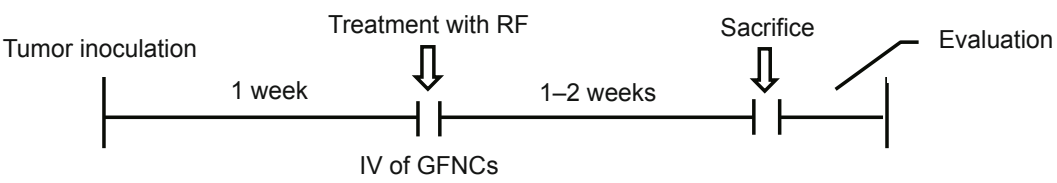

b
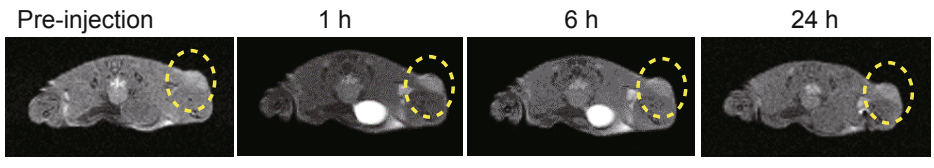

GFNCs+RF
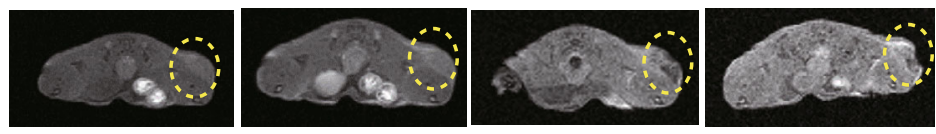

Control
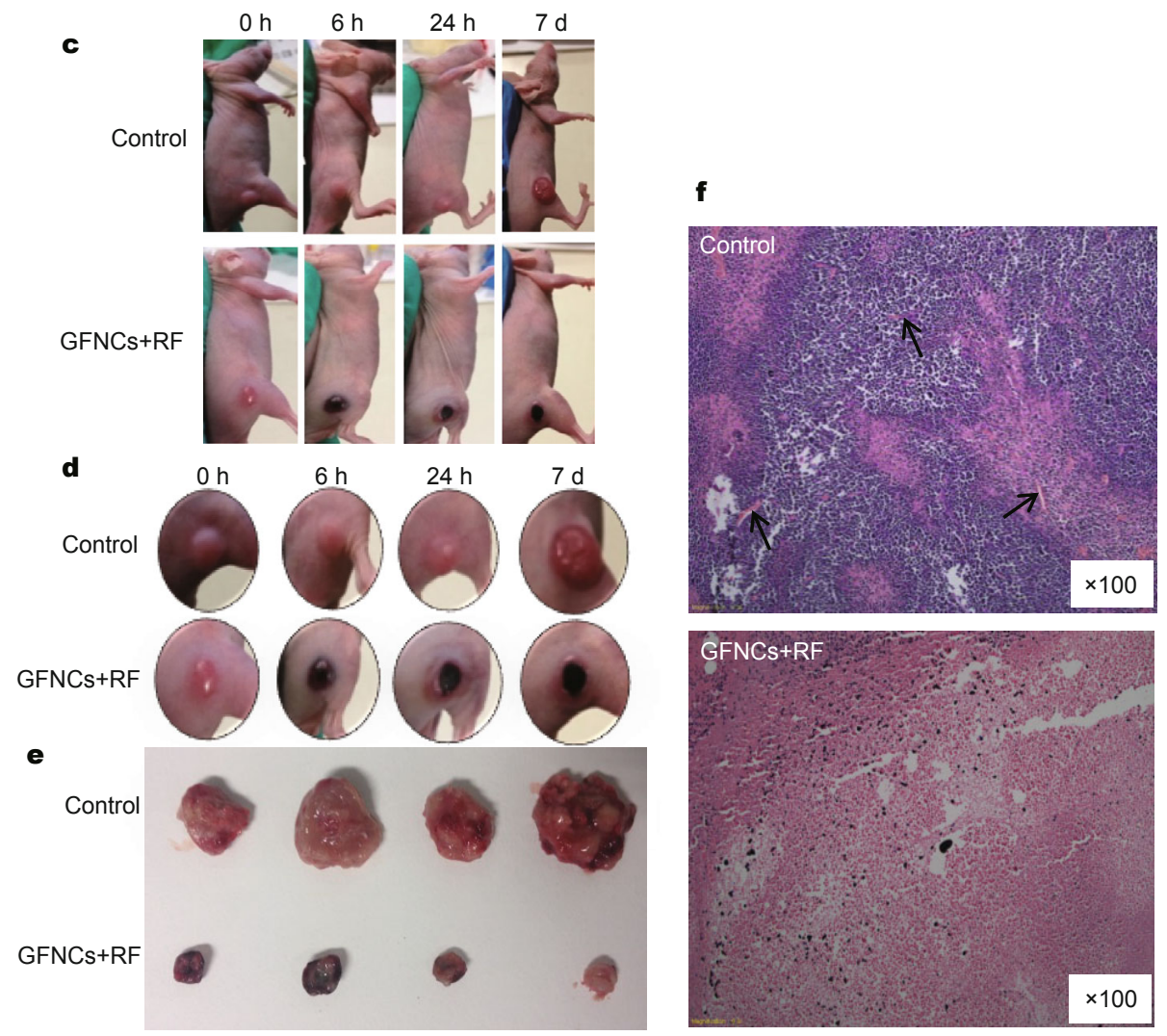

Figure 6 Antitumor effects in vivo for liver cancer by RF-assisted GFNCs treatments. (a) The outline of tumor treatment procedure. (b) $\mathrm{T}_{1}$-weighted MRI of tumors (marked by the dotted circle) at pre- and post- injection of GFNCs, where the commercial Gd-DTPA was used as control. (c) Photographs of nude mice xenografted with H22 liver cancer after GFNCs and RF treatments. (d) The tumors change color from the center to the edge by ischemia and shrank dramatically after $24 \mathrm{~h}$ treatment, while the control group shows no change at all. (e) The images of long-term observation of tumor at $15 \mathrm{~d}$ after treatments. The tumors were entirely necrosis only remaining a crustlike scab compared with the control groups with huge tumor mass. (f) Liver tumor H\&E staining of control group and therapy group one week after the therapy. The tumors treated with RF-assisted GFNCs were hemorrhagic necrosis due to the disrupting of tumor vasculature $(\times 100$ magnification), whereas the control groups are with intact blood vessel (as arrows indicated) and proliferous tumor cells.

the huge tumor mass of control groups (Fig. 6e).

Fig. $6 \mathrm{f}$ showed the H\&E stained tumor slices for the RF-assisted GFNCs treated and control groups. We could observe the ubiquitous blood vessels and dense of packed neoplastic cell with clear tumor proliferation in control groups, whereas, granulation tissues instead of mitotic tumor cells appear in the groups treated by the RF-assisted GFNCs. This indicates that nearly the entire tumor tissues have been severely damaged because of the shutdown of their nutrition supply systems. 


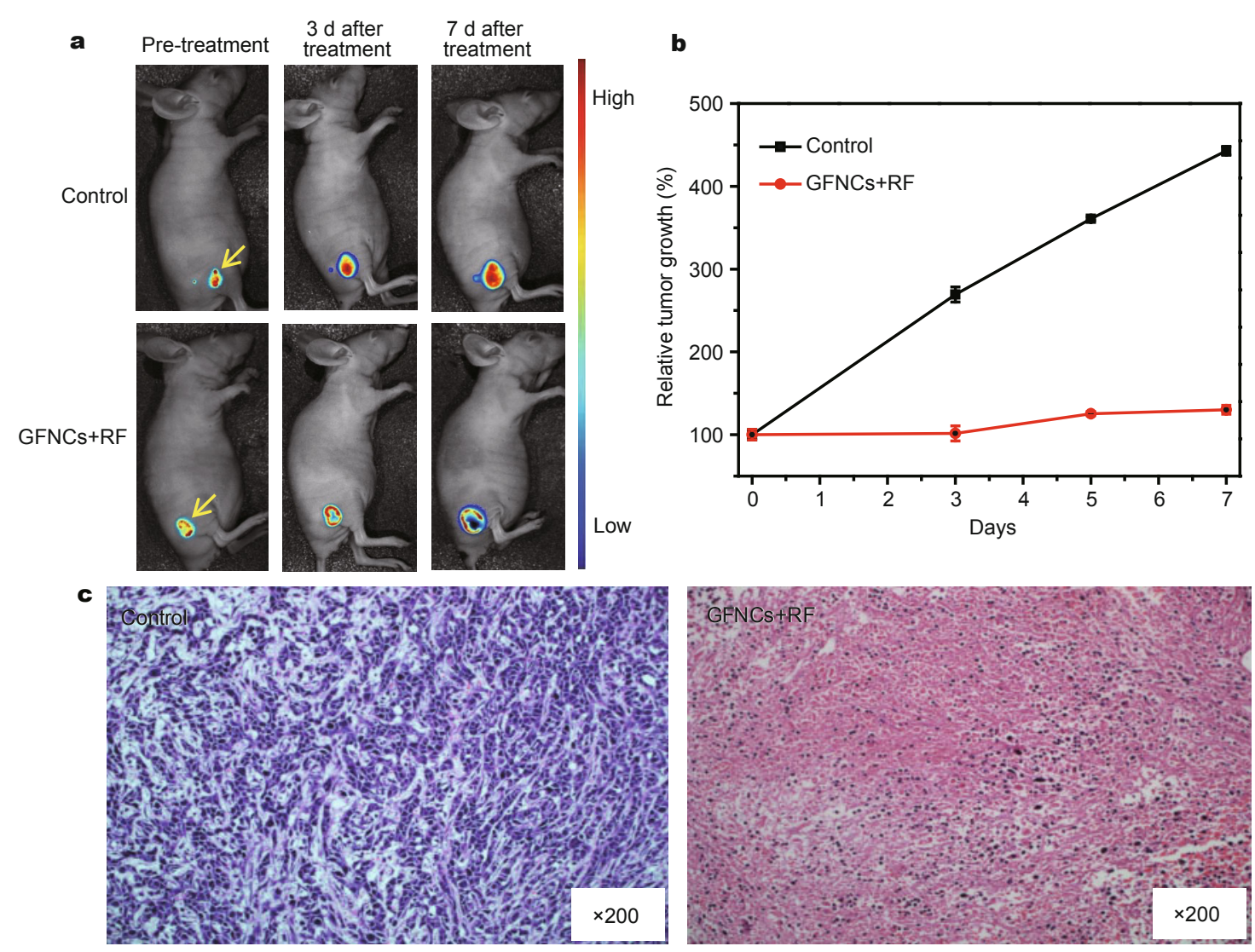

Figure 7 Anticancer therapy in 4T1 cancer model observed by bioluminescence. (a) Integrated bioluminescence emission in 10 min post of the injection of D-luciferin of breast cancer-bearing nude mice with RF-assisted GFNCs treatments using saline as control (yellow arrows labeled tumors). (b) Breast tumor relative growth of therapy group and that of control group with saline, characterized quantitatively by the intensity of bioluminescent of tumor cells. (c) Breast tumor H\&E staining of control group and therapy group one week after the therapy ( $\times 200$ magnification).

The liver tumor is a representative of the cellular tumor, while the breast tumor can represent the desmoplastic tumor. With the same protocol, the extensive necrosis of the breast cancer was also observed based on bioluminescence measurements as well as the histopathological assay (Figs $7 \mathrm{a}-\mathrm{c})$. The proposed new antitumor technique thus may be extended to other types of solid tumors.

\section{Safety evaluation of the antitumor technique}

It was observed that the water soluble microscopic particles of fullerenes and metallofullerenes can be eliminated from the tested rice within several days, as revealed by the MRI measurements (Figs S8a-d) and ${ }^{131}$ I radiolabelling method (Fig. 8), thus it avoids a long-term toxicity of the materials. Moreover, even though there are trace amount of remaining GFNCs, it is known from previous studies that the $\mathrm{Gd} @ \mathrm{C}_{82}$ derivatives may exhibit long-term antitumor activity by inhibiting the tumor growth [33-35]. In fact, the low toxicity in vivo after the therapy has been further verified through tissue histology and analysis, which shows negligible toxicity towards normal tissues. Brain, heart, liver, spleen, lung and kidney slices were collected from the tested mice one week after the therapy, and little visible injuries could be seen in comparison with the control groups (Fig. 9a).

The blood taken from the eyeball of the tested mice one week after the treatment was also analyzed (Fig. 9b), showing minor negative effects on the liver functions. It is noted that the ratio for AST/ALT of GFNCs groups was 4.1 \pm 0.3 , which is much lower than that for the control groups with $6.1 \pm 0.1$, which indicating that the treatments were not causing acute toxicity towards liver. Furthermore, the level of BUN, Cr and Ua showed no obvious fluctuation, suggesting little nephrotoxicity of this material.

\section{DISCUSSION}

Finally, a group of ten liver cancer-bearing normal BAL$\mathrm{B} / \mathrm{c}$ mice were treated following a standard procedure, i.e., the mice were first inoculated with liver cancer, and after 7 


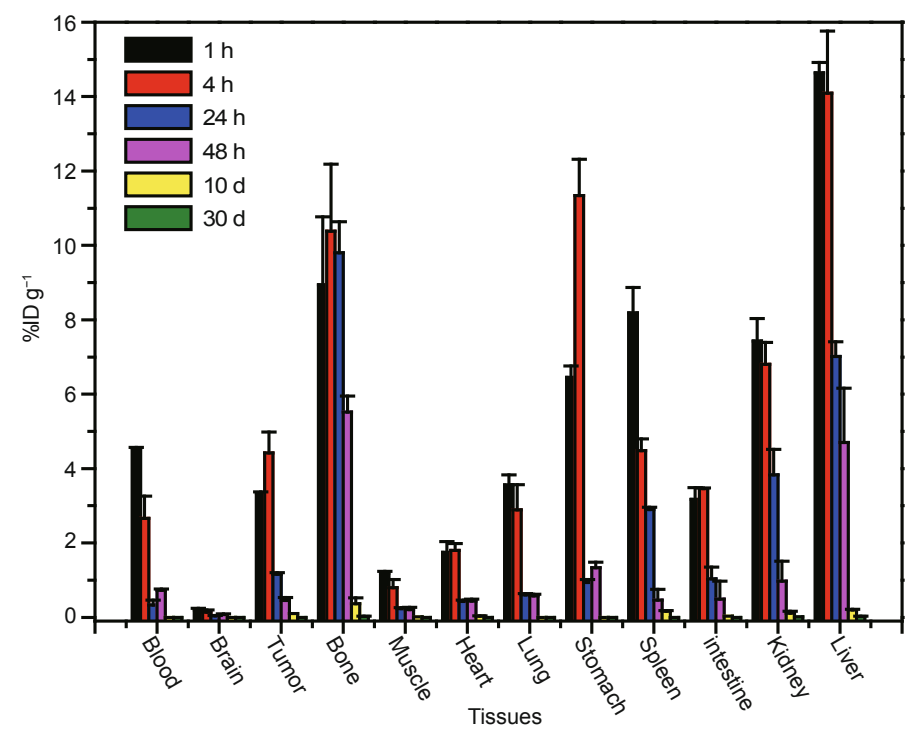

Figure 8 The biodistribution of GFNCs in vivo. GFNCs were IV injected into H22 liver tumor-bearing mice and measured by ${ }^{131}$ I radiolabeled method, $\% \mathrm{ID} / \mathrm{g} \pm$ standard deviation, $n=5$.
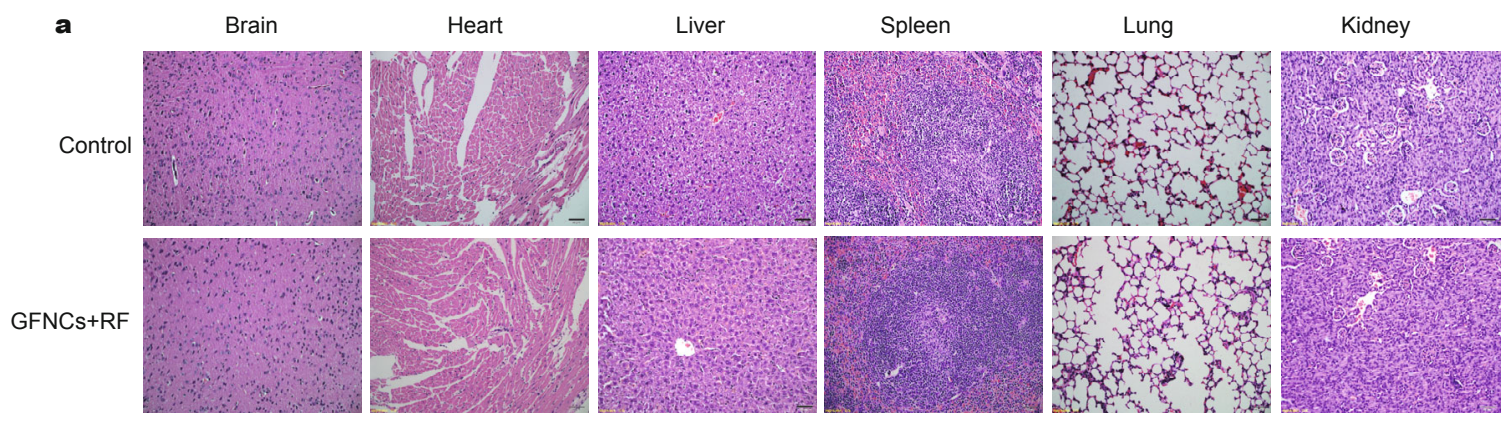

b
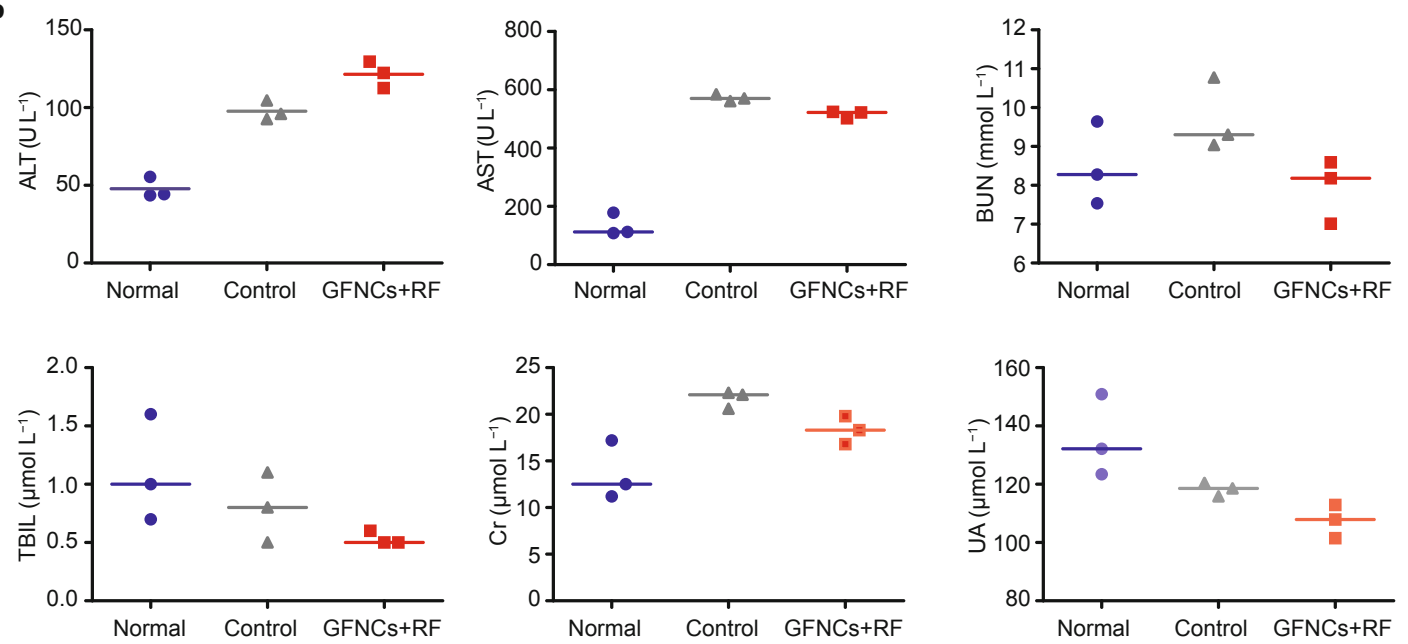

Figure 9 Tissue histology and biochemical analysis. (a) Brain, heart, liver, spleen, lung and kidney are collected from liver tumor-bearing nude mice one week after the treatment, showing negligible toxicities to the normal tissues, $\times 200$ magnification. (b) Serum ALT, AST, BUN, TBIL, Cr and UA levels in normal nude mice and liver tumor-bearing nude mice associated with RF-assisted GFNCs treatments. 
days tumor growth, each mouse was IV injected with 150 $\mu \mathrm{L}$ of $10 \mathrm{mmol} \mathrm{L}^{-1}$ water soluble GFNCs, and then applied the $200 \mathrm{MHz}$ RF radiation for ca. $30 \mathrm{~min}$. All the ten tested mice were observed to show extensive tumor necrosis within a few hours, accompanying with the tumor color changes from pink to black, and finally the collapse of the whole tumor (Fig. S9). The ten as-treated mice were further examined 10 days after the therapy, nearly all of the mice tumors exhibit excellent therapeutic effects, and some of them have been cured after decrustation (Fig. S10). The extremely high performance of this therapeutic technique might be benefited from the activation of the immune system in organism after tumor cell necrosis.

The remarkable performance of this new tumor targeting physiotherapy is overwhelming. It relies on an entire new approach to physically destroy the solid tumors by effectively cutting off their blood supply, which can also significantly reduce toxicity and avoid harmful side effects. Although this technique only works the best for solid tumors, its immediate use will undoubtedly have significant impact to our society. It paves the way to effectively apply the unique properties of advanced nanomaterials for real medical applications.

Received 19 September 2015; accepted 20 September 2015; published online 30 September 2015

1 Nagy JA, Chang SH, Dvorak AM, Dvorak HF. Why are tumour blood vessels abnormal and why is it important to know? Br J Cancer, 2009, 100: 865-869

2 Barinaga M. Designing therapies that target tumor blood vessels. Science, 1997, 275: 482-484

3 Heath VL, Bicknell R. Anticancer strategies involving the vasculature. Nat Rev Clin Oncol, 2009, 6: 395-404

4 Tozer GM, Kanthou C, Baguley BC. Disrupting tumour blood vessels. Nat Rev Cancer, 2005, 5: 423-435

5 Jain RK. Normalization of tumor vasculature: an emerging concept in antiangiogenic therapy. Science, 2005, 307: 58-62

6 Ferrari M. Cancer nanotechnology: opportunities and challenges. Nat Rev Cancer, 2005, 5: 161-171

7 Kievit FM, Zhang M. Cancer nanotheranostics: improving imaging and therapy by targeted delivery across biological barriers. Adv Mater, 2011, 23: H217-H247

8 Greish K. Enhanced permeability and retention of macromolecular drugs in solid tumors: a royal gate for targeted anticancer nanomedicines. J Drug Target, 2007, 15: 457-464

9 Fang J, Nakamura H, Maeda H. The EPR effect: unique features of tumor blood vessels for drug delivery, factors involved, and limitations and augmentation of the effect. Adv Drug Deliv Rev, 2011, 63: 136-151

10 Liu X, Chen Y, Li H, et al. Enhanced retention and cellular uptake of nanoparticles in tumors by controlling their aggregation behavior. ACS Nano, 2013, 7: 6244-6257

11 Thakor AS, Gambhir SS. Nanooncology: the future of cancer diagnosis and therapy. CA-Cancer J Clin, 2013, 63: 395-418

12 Li Y, Lin TY, Luo Y, et al. A smart and versatile theranostic nano- medicine platform based on nanoporphyrin. Nat Commun, 2014, 5: 4712

13 Siemann DW, Horsman MR. Vascular targeted therapies in oncology. Cell Tissue Res, 2009, 335: 241-248

14 Wang M, Thanou M. Targeting nanoparticles to cancer. Pharm Res, 2010, 62: 90-99.

15 de Bono JS, Ashworth A. Translating cancer research into targeted therapeutics. Nature, 2010, 467: 543-549

16 Shu CY, Ma XY, Zhang JF, et al. Conjugation of a water-soluble gadolinium endohedral fulleride with an antibody as a magnetic resonance imaging contrast agent. Bioconjugate Chem, 2008, 19: 651-655

17 Shu C, Corwin FD, Zhang J, et al. Facile preparation of a new gadofullerene-based magnetic resonance imaging contrast agent with high ${ }^{1} \mathrm{H}$ relaxivity. Bioconjugate Chem, 2009, 20: 1186-1193

18 Guo YG, Wan LJ, Li CJ, et al. The effects of annealing on the structures and electrical conductivities of fullerene-derived nanowires. J Mater Chem, 2004, 14: 914-918

19 Wang CR, Kai T, Tomiyama T, et al. Materials science: $\mathrm{C}_{66}$ fullerene encaging a scandium dimer. Nature, 2000, 408: 426-427

20 Wang $\mathrm{T}, \mathrm{Wu} \mathrm{J}, \mathrm{Xu} \mathrm{W}$, et al. Spin divergence induced by exohedral modification: ESR study of $\mathrm{Sc}_{3} \mathrm{C}_{2} @ \mathrm{C}_{80}$ fulleropyrrolidine. Angew Chem Int Ed, 2010, 49: 1786-1789

21 Li CJ, Guo YG, Li BS, et al. Template synthesis of $\mathrm{Sc}_{0} \mathrm{C}_{82}(\mathrm{I})$ nanowires and nanotubes at room temperature. Adv Mater, 2005, 17: $71-73$

22 Sitharaman B, Bolskar RD, Rusakova I, Wilson LJ. Gd@C 60 $\left[\mathrm{C}(\mathrm{COOH})_{2}\right]_{10}$ and $\mathrm{Gd} @ \mathrm{C}_{60}(\mathrm{OH})_{x}$ : nanoscale aggregation studies of two metallofullerene MRI contrast agents in aqueous solution. Nano Lett, 2004, 4: 2373-2378

23 Shu CY, Wang CR, Zhang JF, et al. Organophosphonate functionalized $\mathrm{Gd} @ \mathrm{C}_{82}$ as a magnetic resonance imaging contrast agent. Chem Mater, 2008, 20: 2106-2109

24 Husebo LO, Sitharaman B, Furukawa K, Kato T, Wilson LJ. Fullerenols revisited as stable radical anions. J Am Chem Soc, 2004, 126: 12055-12064

25 Zheng JP, Zhen MM, Ge JC, et al. Multifunctional gadofulleride nanoprobe for magnetic resonance imaging/fluorescent dual modality molecular imaging and free radical scavenging. Carbon, 2013, 65: 175-180

26 Di Corato R, Béalle G, Kolosnjaj-Tabi J, et al. Combining magnetic hyperthermia and photodynamic therapy for tumor ablation with photoresponsive magnetic liposomes. ACS Nano, 2015, 9: 29042916

27 Tamarov KP, Osminkina LA, Zinovyev SV, et al. Radio frequency radiation-induced hyperthermia using Si nanoparticle-based sensitizers for mild cancer therapy. Sci Rep, 2014, 4: 7034

28 Chu KF, Dupuy DE. Thermal ablation of tumours: biological mechanisms and advances in therapy. Nat Rev Cancer, 2014, 14: 199-208

29 Moran C, Wainerdi S, Cherukuri T, et al. Size-dependent Joule heating of gold nanoparticles using capacitively coupled radiofrequency fields. Nano Res, 2009, 2: 400-405

30 Gaya AM, Rustin GJS. Vascular disrupting agents: a new class of drug in cancer therapy. Clin Oncol, 2005, 17: 277-290

31 Hinnen P, Eskens FALM. Vascular disrupting agents in clinical development. Br J Cancer, 2007, 96: 1159-1165

32 Bhave M, Akhter N, Rosen ST. Cardiovascular toxicity of biologic agents for cancer therapy. Oncology-NY, 2014, 28: 482-490

33 Chen C, Xing G, Wang J, et al. Multihydroxylated [Gd@ $\left.\mathrm{C}_{82}(\mathrm{OH})_{22}\right]_{n}$ nanoparticles: antineoplastic activity of high efficiency and low toxicity. Nano Lett, 2005, 5: 2050-2057

34 Yang D, Zhao Y, Guo H, et al. $\left[\mathrm{Gd} @ \mathrm{C}_{82}(\mathrm{OH})_{22}\right]_{n}$ nanoparticles induce dendritic cell maturation and activate Th1 immune responses. 
ACS Nano, 2010, 4: 1178-1186

35 Kang SG, Zhou G, Yang P, et al. Molecular mechanism of pancreatic tumor metastasis inhibition by $\mathrm{Gd} @ \mathrm{C}_{82}(\mathrm{OH})_{22}$ and its implication for de novo design of nanomedicine. Proc Natl Acad Sci USA, 2012, 109: 15431-15436

Acknowledgments This work was supported by the National Natural Science Foundation of China (51472248, 11179006 and 51372251) and the Key Research Program of the Chinese Academy of Sciences (KGZDEW-T02 and XDA09030302). We thank Prof. Yan Li of Zhongnan Hospital of Wuhan University for help with tissues histology and biochemical analysis. We also thank Yongtao Li, Zhentao Zuo, and Yuqing Wang for developing the RF set-ups.
Author contributions Wang $\mathrm{C}$ and Zhen M supervised the project and designed the experiments. Li J synthesized the GFNCs. Zhen M and Zhang G performed the in vivo experiments. Zhen M, Deng R and Zou T performed the in vitro characterization. Shu $\mathrm{C}$ and Wang $\mathrm{T}$ analyzed the nature of GFNCs. Fang F and Lei $\mathrm{H}$ performed the animal MRI studies. Wang C, Bai C and Luo Y contributed to the interpretation of the data. Zhen M, Luo $\mathrm{Y}$ and Wang $\mathrm{C}$ wrote the manuscript.

Conflict of interests The authors declare that they have no conflict of interest.

Supplementary information Supporting data are available in the online version of the paper.

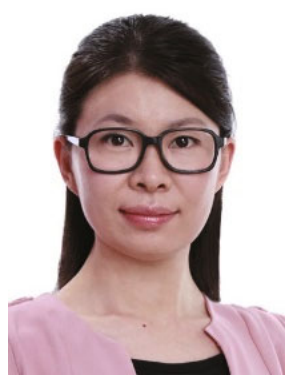

Mingming Zhen was born in 1987. She received her PhD degree in physical chemistry from the Institute of Chemistry, Chinese Academy of Sciences (ICCAS) in 2014. Currently, she is an assistant professor at ICCAS. Her research interests include biomedical applications of fullerenes and gadofullerenes.

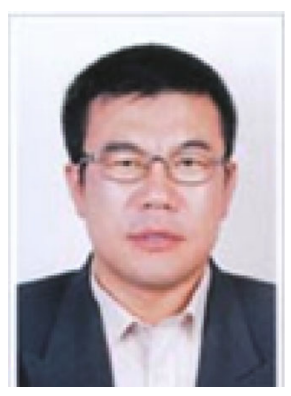

Chunru Wang was born in 1965. He received his PhD degree in physical chemistry from Dalian Institute of Chemistry Physics, Chinese Academy of Sciences in 1992. Currently, he is a professor at ICCAS. His research interests include fullerenes and endohedral fullerenes, mainly focusing on their industrialization and applications. He discovered the metal carbide fullerenes for the first time, researched on high efficiency MRI contrast agents and developed a novel tumor vascular-targeting therapy technique using gadofullerenes.

中文摘要 本文报道了一种利用金属富勒烯纳米晶体快速高效治疗肿瘤的新技术. 从生物学上肿瘤血管和正常血管在结构上存在显著 差异这一特点着手, 利用材料学上金属富勒烯纳米晶体在吸收射频能量后发生相变, 伴随着体积剧烈膨胀的特性, 高选择性地摧毁肿 瘤血管. 研究表明, 经过 1小时治疗后, 肿瘤部位血流即可发生快速阻断, 治疗 2 4 小时后, 肿瘤组织逐步发生出血性坏死, 肿瘤塌陷体积 缩小; 并且对于多种实体肿瘤均有显著疗效. 该技术是一种快速、广谱、特异性高、毒副作用小的新型肿瘤治疗技术, 是一种具有巨 大发展潜力的肿瘤治疗技术. 